\title{
A Novel Method for Transmission Electron Microscopy Study of Cytoplasmic Fragments From Preimplantation Human Embryos
}

\author{
IMAN HALVAEI, ${ }^{1}$ MOHAMMAD ALI KHALILI ${ }^{1} *$ AND STEFANIA A NOTTOLA ${ }^{2}$ \\ ${ }^{1}$ Research and Clinical Center for Infertility, Shahid Sadoughi University of Medical Sciences, Yazd, Iran \\ ${ }^{2}$ Department of Anatomy, Histology, Forensic Medicine and Orthopaedics, La Sapienza University of Rome, Rome, Italy
}

\author{
KEYWORDS Transmission electron microscopy; embryo; fragmentation; empty zona pellucida
}

\begin{abstract}
Transmission electron microscopy (TEM) is the main tool for exploring the intracellular damage and organelle distribution. The cause of producing embryo cytoplsamic fragmentation is not completely understood. Since the fragments have detrimental effects on embryo development, the ultrastructural analysis of fragments may play an important role in fragmentation etiology and in embryo development as well. There are no studies regarding the ultrastructure of fragments in transferable embryos, because the preparation for TEM is not vital and embryos are discarded inevitably. This study aims to introduce a new method for ultrastructural evaluation of fragments without damaging the human cleaving embryos. Microsc. Res. Tech. 00:000-000, 2016. @ 2016 Wiley Periodicals, Inc.
\end{abstract}

\section{INTRODUCTION}

Both scanning electron microscopy (SEM) and transmission electron microscopy (TEM) are the best tools for ultrastructural evaluation of the cells. However, the high technology TEM provides accurate and detailed data from inside the cells. These include cell damage, distribution of organelles and abnormalities regarding their fine morphology that introduces this technique as a first line investigation tool in this era (Khalili et al., 2012, 2013; Nottola et al., 2009). In addition, TEM detects occurrence of apoptosis inside the cells (Tilly, 1996). Evaluation of fine morphology in human embryos provides valuable information regarding size and number of organelles, distribution and association of fragments with neighboring blastomeres, and blastomeres junctions. Also, embryo development can be predictable from ultrastructural changes (Familiari et al., 2006; Nottola et al., 2000).

Embryos in in vitro fertilization (IVF) units are selected for embryo transfer (ET) mainly based on their morphological features. Numerous embryo scoring systems have been introduced in last decades that the grade of fragmentation is considered as the main morphological features in scoring (Lundin and Ahlström, 2015). Some studies are in favor of detrimental effect of fragments on subsequent embryo development. It was reported that high fragmentation (>15\% (Alikani et al., 2000), >25\% (Hardy et al., 2003) of preimplantation embryos negatively affects blastocyst hatching and embryo implantation. Another negative effect of fragments is the impact on blastomere volume, since they occupy the space inside the zona pellucida (ZP), which has been shown that increasing fragmentation will decrease mean blastomere volume (Hnida et al., 2004).
The etiology of generating fragments is not totally understood. Fujimoto et al. (2011) reviewed some hypotheses on the origin of fragments including: role of telomere length, maternal age, role of reactive oxygen species, and association with lipoprotein levels. The source of fragmentation could be determined using TEM. For example, TEM distinguishes whether fragmentation is related to apoptosis or is a physiological process or how the fragments change with increasing maternal age. In order to evaluate the fragments at TEM level, the embryo should be fixed, which makes it unsuitable for ET. Therefore, the main goal was introducing a novel technique for ultrastructural assessment of fragments, without interrupting the embryo viability.

\section{MATERIALS AND METHODS Preparation of Micro-Pipettes}

Micro-pipettes with different sizes were prepared for this method. The borosilicate glass capillaries (Drummond, USA) with outer diameter of $0.97 \mathrm{~mm}$ and inner diameter of $0.69 \mathrm{~mm}$ were pulled, first. After washing the capillaries with milli-Q water, they were pulled by horizontal puller (Sutter Instrument, USA). For making fragment removal micro-pipette, the micro-pipette was bent by heating of microforge (MF9, Narishige, Japan) to an angle of $35-40^{\circ}$, with inner diameter of $12-15 \mu \mathrm{m}$. The same procedure was performed for preparation of micro-pipette used for aspiration of ZP content, except the inner diameter was 20-30 $\mu \mathrm{m}$. For preparation of holding pipette, it was heated by

\footnotetext{
*Correspondence to: Mohammad Ali Khalili; Safayeh, Bou-ali ave., Yazd 8916877391, Iran. E-mail: khalili59@hotmail.com

Received 9 January 2016; accepted in revised form 20 February 2016

REVIEW EDITOR: Prof. George Perry

DOI 10.1002/jemt.22657

Published online 00 Month 2016 in Wiley Online Library (wileyonlinelibrary.com).
} 

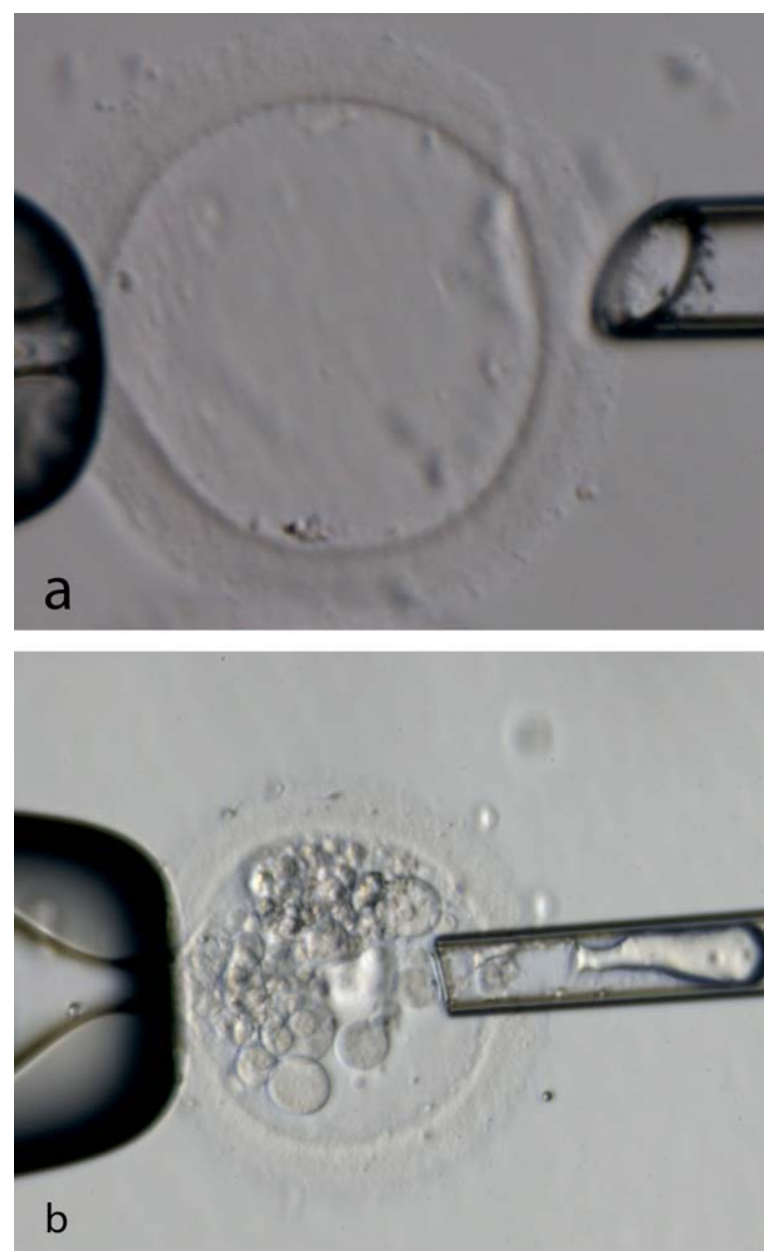

Fig. 1. Different steps in preparation of fragments before fixation for TEM. Unfertilized oocytes or discarded embryos are used for preparation of empty $\mathrm{ZP}(\mathrm{a})$, then the selected fragments that are removed microsurgically, are put inside the empty ZP (b). [Color figure can be viewed in the online issue, which is available at wileyonlinelibrary. com.]

microforge after pulling as mentioned above. The inner, outer, and opening diameters were 20-30, 120, and $3-5 \mu \mathrm{m}$, respectively. After preparation, all micropipettes were sterilized by dry heat at $120^{\circ} \mathrm{C}$ for $6 \mathrm{~h}$.

\section{Polyvinyl Pyrrolidone 30\%}

The fragment removal pipette was filled by Polyvinyl Pyrrolidone (PVP) 30\% for making better manipulation and suction of fragments. Three grams of PVP was mixed with MOPS media (Vitrolife, Sweden), while the total volume was reached to $10 \mathrm{~mL}$. After preparation, it was kept at $4^{\circ} \mathrm{C}$ until use.

\section{Preparation of Suitable Dish}

Calcium and magnesium ( $\mathrm{Ca}-\mathrm{Mg}$ ) free medium (Vitrolife, Sweden) supplemented with 10\% human serum albumin (Vitrolife, Sweden) was selected as a medium for fragment removal. One micro-drop of PVP $30 \%$ was placed in the middle of Falcon 1006 petri dish (Falcon, USA) and eight micro-drops of $\mathrm{Ca}-\mathrm{Mg}$ free medium were placed around the PVP. The drops were overlaid by mineral oil (Vitrolife, Sweden) and kept at $37^{\circ} \mathrm{C}$.

\section{Preparation of Empty ZP}

Unfertilized oocytes or discarded embryos were used for making empty ZP. First, a petri dish was prepared. After setting specific home-made pipette (inner diameter: $20-30 \mu \mathrm{m})$ and holding pipette, the pipette was filled with PVP $30 \%$; then, the mineral oil was drawn into the pipette. Finally, the oocyte/embryo was placed in $\mathrm{Ca}-\mathrm{Mg}$ free medium. After several minutes, the oocyte/embryo was held by holding pipette and ZP was hatched using laser beam. Then, the pipette was inserted into the oocyte/embryo and the ooplasm/blastomeres were suctioned gently. This process was continued until the ZP was completely empty (Fig. 1).

\section{Fragment Removal}

The embryo selected for TEM evaluation of its fragments, was first incubated in $\mathrm{Ca}-\mathrm{Mg}$ free media. After filling the fragment removal pipette with PVP, the embryo was held with holding pipette with consideration of best orientation of fragments. The embryo was hatched using $1480 \mathrm{~nm}$ wave length infrared diode laser for $2 \mathrm{~ms}$ duration to open a $10-12 \mu \mathrm{m}$ hole in $\mathrm{ZP}$. The fragment removal pipette was gently entered close to the fragments to remove them by light suction. Immediately after fragment removal, the embryo was carefully washed in embryo culture media and was transferred to incubator for further culture. The removed fragments were entered empty ZP by fragment removal pipette (Fig. 1).

\section{TEM Procedure}

Fixation. The fragments entrapped in the empty ZP were fixed by glutaraldehyde $2.5 \%$ diluted by cacodylate buffer $0.2 \mathrm{M}$. Then, they were transferred to central well (Falcon, USA) containing $500 \mu \mathrm{L}$ glutaraldehyde and kept at $4^{\circ} \mathrm{C}$ for $1 \mathrm{~h}$. The samples were then washed in cacodylate buffer for 10 min three times at room temperature. For Secondary fixation, the samples were transferred to central well containing $500 \mu \mathrm{L}$ osmium tetroxide $1 \%$ and kept at $4^{\circ} \mathrm{C}$ for $1 \mathrm{~h}$. The specimens were then rinsed in cacodylate buffer for 10 min three times. The washing step was followed by rinse in deionized water for 10 min twice.

Agarose Embedding. The sample was precisely placed in the center of warm agar using handler and $140 \mu \mathrm{m}$ diameter pipette. The slide was kept in the refrigerator for $20 \mathrm{~min}$ to make the agar hard. Then, the excess agar was cut to a cube of $1 \times 1 \times 1 \mathrm{~mm}$ containing the sample in the center.

Dehydration. Dehydration process was followed by ethylic alcohol as follows: alcohol ethylic 30\%, 5-10 min, alcohol ethylic 50\%, 5-10 min, alcohol ethylic 70\% 5-10 min, alcohol ethylic 80\% 5-10 min, alcohol ethylic 90\% 5-10 min, alcohol ethylic 100\% 5-10 min (two times).

Resin Infiltration. Resin infiltration was performed as follows: resin/ethanol $100 \%(1 / 3) 1 \mathrm{~h}$, resin/ ethanol $100 \%(1 / 1) 1 \mathrm{~h}$, resin/ethanol $100 \%(3 / 1) 2 \mathrm{~h}$, pure resin 12-16 h, and pure resin $3 \mathrm{~h}$.

Embedding. First, the label for each sample was placed in mold impregnated to resin and the mold was placed in the oven $\left(60^{\circ} \mathrm{C}\right)$ for $3 \mathrm{~h}$. Thereafter, the resin 

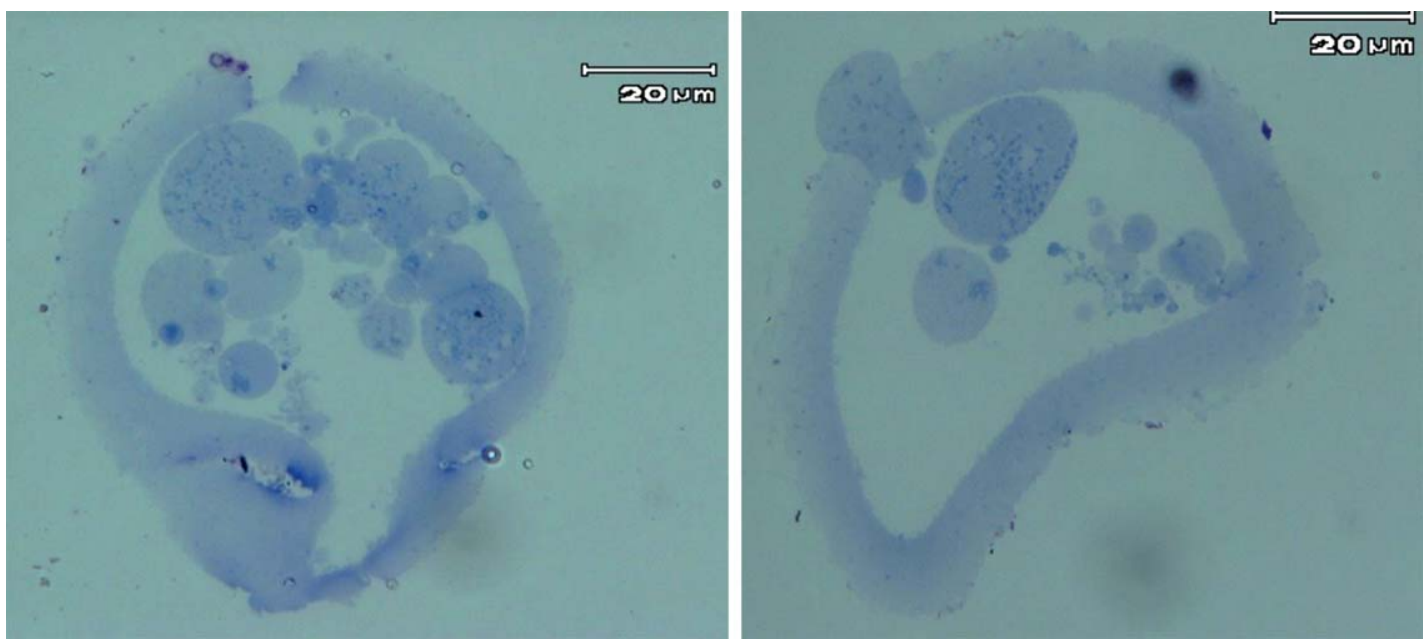

Fig. 2. Light microscopy of semithin section after toluidine blue staining from two different blocks. Note, the presence of fragments that are pulled inside the empty ZP $(\times 1000)$. [Color figure can be viewed in the online issue, which is available at wileyonlinelibrary.com.]

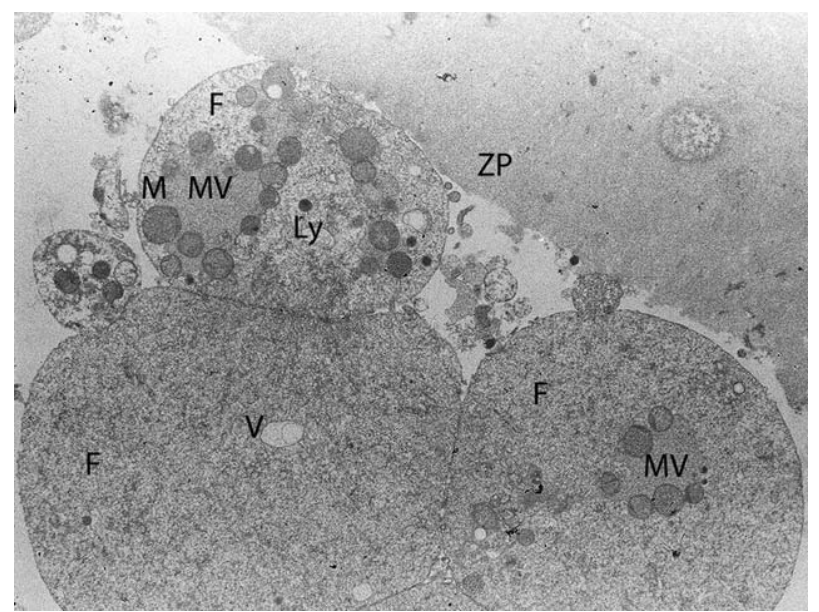

Fig. 3. The morphology and organelle microtopography of embryo fragments inside the empty ZP by TEM. F: fragment; ZP: zona pellucida; M: mitochondrium; MV: mitochondria-vesicle; Ly: primarylysosome; V: vacuole $(\times 4400)$.

was gently poured in molds until the half of models were filled. The sample was precisely placed in the top of mold by a needle. The molds were then transferred into oven $\left(60^{\circ} \mathrm{C}\right)$ for $2 \mathrm{~h}$. After that, the molds were filled with pure resin and placed in the oven for at least $48 \mathrm{~h}$ for resin polymerization.

Sectioning. The semithin sections $(500 \mathrm{~nm})$ were prepared by a glass knife using Ultra-cut UCT microscope (Leica Microsystems, Germany). The sections were stained with toluidine blue to check the samples in the sections (Fig. 2). After reaching to samples, ultrathin sections of 50-70 $\mathrm{nm}$ were prepared using a diamond knife. Then, the samples were placed on copper grids. The grids were then checked with TEM (Zeiss, Germany).

\section{Staining and Observation}

Heavy metals salts were used for samples prepared for TEM. Two main stains that were used prior to
TEM observation were uranyl acetate and lead citrate. The uranyl ions bind to phosphate and amino groups which are present in the nucleic acids and proteins. The lead ions attach to compound with negative charge and structures that are osmium-reacted like membranes. The staining steps were performed as follows: uranyl acetate $60 \mathrm{~min}$, distilled water 1-2 min, lead citrate $60 \mathrm{~min}$, and distilled water 1-2 min. For TEM imaging, the grids were left in the room temperature to dry. Then the grids were placed in the electron microscope chamber to observe.

\section{RESULTS}

The TEM micrographs showed the fragments have distinct membrane with various cytoplasmic organelles. These were large vacuoles, large mitochondriavesicle (MV) complexes, primary lysosomes, and mitochondria (Fig. 3).

\section{DISCUSSION}

Along with the advantages of TEM, there are some limitations including: high expense of this technology, requiring highly trained personnel, and sample fixation for ultra-observation (Khalili et al., 2012). The later limitation leads to discarding the embryos from ART program. However, by the method introduced in this study, the embryos could be preserved for the patients, as only the fragments are removed for TEM assessment.

Formation of high quality embryos is one of the main goals in IVF clinics. There have been numerous studies regarding the inverse relationship between degree of fragmentation and embryo viability (Fujimoto et al., 2011). Also, the degree of embryo fragmentation could be predictive factor for live birth (Rhenman et al., 2015). There are, however, some hypotheses related to the embryo itself including: abnormal cytoplasmic adenosine triphosphate content, role of apoptosis, relation to the progress of the meiotic and the mitotic cell cycles, and cytoskeleton/microtubule organization status (Alikani, 2005; Mateusen 
et al., 2005; Stensen et al., 2015; Van Blerkom et al., 2001). Evaluation of fragments ultrastructure can throw light on understanding the main mechanism of fragmentation.

Some studies evaluated ultrastructure of human preimplantation embryos that used donated surplus embryos, polyspermic or arrested embryos (Au et al., 2005; Chi et al., 2011; Pereda and Croxatto, 1978; Makabe et al., 2000; Motta et al., 1988, 2000; Nottola et al., 2000; Pereda et al., 1999; Van Blerkom et al., 2001;). IVF clinic usually discards unfertilized oocytes or heavy fragmented embryos, which can be used as carriers for evaluating fragments ultrastructurally. Our method has several advantages: (a) the defragmented embryo still can be used for the patient, (b) the fragments in low grade embryos can be evaluated, and (c) TEM assessment of fragments in certain cases, like the impact of different conditions and stimulating drugs on fine morphology of fragments, or in the patients with low number of embryos. Another advantage of this method is the possibility of comparison between ultrastructural changes and IVF outcomes. Because, the embryo that its fragments were evaluated ultrastructurally, could be transferred and rates of abortion, pregnancy, congenital anomaly, and live birth might be compared with TEM results. Regarding the safety of embryo defragmentation, many scientists have successfully performed it previously (Alikani et al., 1999; Halvaei et al., 2015; Keltz et al., 2010). In conclusion, for the first time, we report the novel technique of fragments housing inside the empty ZP for ultrastructure study of fragments without disturbing the embryo viability. This method can be also used for evaluation of other biological samples, like debris, granules, and sperm cells.

\section{ACKNOWLEDGMENT}

This study was extracted from Ph.D. thesis of Iman Halvaei. The authors appreciate Drs. Shahin Ghzali and Azam Agha-Rahimi for their valuable helps during the study. The authors would like to thank Yazd Research and Clinical Center for Infertility for providing the financial support for performing this study.

\section{CONFLICT OF INTEREST}

Authors do not have any conflict of interest.

\section{REFERENCES}

Alikani M. 2005. Epithelial cadherin distribution in abnormal human pre-implantation embryos. Hum Reprod 20:3369-3375.

Alikani M, Calderon G, Tomkin G, Garrisi J, Kokot M, Cohen J. 2000. Cleavage anomalies in early human embryos and survival after prolonged culture in-vitro. Hum Reprod 15:2634-2643.

Alikani M, Cohen J, Tomkin G, Garrisi GJ, Mack C, Scott RT. 1999. Human embryo fragmentation in vitro and its implications for pregnancy and implantation. Fertil Steril 71:836-842.

Au H, Yeh T, Kao S, Tzeng C, Hsieh R. 2005. Abnormal mitochondrial structure in human unfertilized oocytes and arrested embryos. Ann N Y Acad Sci 1042:177-185.
Chi H-J, Koo J-J, Choi S-Y, Jeong H-J, Roh S-I. 2011. Fragmentation of embryos is associated with both necrosis and apoptosis. Fertil Steril 96:187-192.

Familiari G, Heyn R, Relucenti M, Nottola SA, Sathananthan AH. 2006. Ultrastructural dynamics of human reproduction, from ovulation to fertilization and early embryo development 1 . Int Rev Cytol 249:53-141.

Fujimoto VY, Browne RW, Bloom MS, Sakkas D, Alikani M. 2011. Pathogenesis, developmental consequences, and clinical correlations of human embryo fragmentation. Fertil Steril 95:1197-1204.

Halvaei I, Khalili MA, Safari S, Esfandiari N. 2015. Ongoing pregnancies following cosmetic micromanipulation of preimplantation embryos in patients with implantation failure. Case Rep Med 2015:

Hardy K, Stark J, Winston RM. 2003. Maintenance of the inner cell mass in human blastocysts from fragmented embryos. Biol Reprod 68:1165-1169.

Hnida C, Engenheiro E, Ziebe S. 2004. Computer-controlled, multilevel, morphometric analysis of blastomere size as biomarker of fragmentation and multinuclearity in human embryos. Hum Reprod 19:288-293.

Keltz M, Fritz R, Gonzales E, Ozensoy S, Skorupski J, Stein D. 2010. Defragmentation of low grade day 3 embryos resulted in sustained reduction in fragmentation, but did not improve compaction or blastulation rates. Fertil Steril 94:2406-2408.

Khalili MA, Maione M, Palmerini MG, Bianchi S, Macchiarelli G, Nottola SA. 2012. Ultrastructure of human mature oocytes after vitrification. Eur J Histochem 56:236-242.

Khalili MA, Nottola SA, Shahedi A, Macchiarelli G. 2013. Contribution of human oocyte architecture to success of in vitro maturation technology. Iran J Reprod Med 11:1-10.

Lundin K, Ahlström A. 2015. Quality control and standardization of embryo morphology scoring and viability markers. Reprod Biomed Online 31:459-471.

Makabe S, Naguro T, Nottola S, Motta P. 2000. Ultrastructural dynamic features of in vitro fertilization in humans. Ital J Anat Embryol 106:11-20.

Mateusen B, Van Soom A, Maes DG, Donnay I, Duchateau L, Lequarre AS. 2005. Porcine embryo development and fragmentation and their relation to apoptotic markers: A cinematographic and confocal laser scanning microscopic study. Reproduction 129: 443-452.

Motta PM, Nottola SA, Makabe S, Heyn R. 2000. Mitochondrial morphology in human fetal and adult female germ cells. Hum Reprod 15:129-147.

Motta PM, Nottola SA, Micara G, Familiari G. 1988. Ultrastructure of human unfertilized oocytes and polyspermic embryos in an IVFET Program. Ann N Y Acad Sci 541:367-383.

Nottola S, Coticchio G, Sciajno R, Gambardella A, Maione M, Macchiarelli G, Borini A. 2009. Ultrastructural changes in human metaphase II oocytes cryopreserved by vitrification. Reprod Biomed Online 19:17-27.

Nottola S, Makabe S, Stallone T, Familiari G, Macchiarelli G, Motta P. 2000. In vitro fertilized human eggs. An electron microscopic study. Ital J Anat Embryol 106:75-83.

Pereda J, Croxatto H. 1978. Ultrastructure of a seven-cell human embryo. Biol Reprod 18:481-489.

Rhenman A, Berglund L, Brodin T, Olovsson M, Milton K, Hadziosmanovic N, Holte J. 2015. Which set of embryo variables is most predictive for live birth? A prospective study in 6252 single embryo transfers to construct an embryo score for the ranking and selection of embryos. Hum Reprod 30:28-36.

Sathananthan A, Tarin J, Gianaroli L, Ng S, Dharmawardena V, Magli M, Fernando R, Trounson A. 1999. Development of the human dispermic embryo. Hum Reprod Update 5:553-560.

Stensen MH, Tanbo TG, Storeng R, AAbyholm T, Fedorcsak P. 2015. Fragmentation of human cleavage-stage embryos is related to the progression through meiotic and mitotic cell cycles. Fertil Steril 103:374-381.

Tilly JL. 1996. Apoptosis and ovarian function. Rev Reprod 1:162172.

Van Blerkom J, Davis P, Alexander S. 2001. A microscopic and biochemical study of fragmentation phenotypes in stage-appropriate human embryos. Hum Reprod 16:719-729. 